\title{
AVALIAÇÃO DA VELOCIDADE ESPECÍFICA DE CRESCIMENTO RADIAL DE FUNGOS EM ÓLEO VEGETAL RESIDUAL
}

\author{
Tania Maria Costa ${ }^{1}$, João Guilherme Costa Sperb ${ }^{2}$, André Lima Roncheti ${ }^{3}$, \\ Tatiani Karini Rensi Botelho ${ }^{4}$, Thais Marly Sell ${ }^{5}$, Sávio Leandro Bertoli ${ }^{6}$, \\ Lorena Benathar Ballod Tavares ${ }^{7}$
}

\begin{abstract}
Resumo: A quantidade de resíduos oleosos gerados diariamente por indústrias de distintas áreas e o descarte inadequado de óleos residuais em ambientes domésticos e em ambientes naturais têm ocasionado preocupação mundial, devido à extensão dos danos ambientais causados por estes poluentes. Distintos fungos apresentam potencial de crescimento quando cultivados em ambientes contendo substratos oleosos. O crescimento micelial radial, bem como a cinética e a velocidade específica de crescimento de fungos filamentosos isolados de resíduos do processamento de óleo vegetal e de petróleo, foram avaliados em placas de Petri contendo óleo de soja virgem ou óleo de soja residual. Dentre os 24 fungos testados, foram identificados os dos gêneros Penicillium, Rhizopus, Aspergillus e Scopulariopsis. Os fungos LEB 025, 029, 033, 039, 040, 042 e 044 apresentaram as velocidades especificas máximas de crescimento estimuladas pela presença de óleo de soja residual, não havendo inibição de crescimento para qualquer estirpe estudada. Estes resultados sugerem que os fungos avaliados neste estudo, podem apresentar potencial para aplicação em processos de biorremediação de ambientes contaminados com óleos residuais, bem como sua utilização na sintese de compostos como enzimas lipolíticas e biossurfactantes.
\end{abstract}

Palavras-chave: Identificação. Cinética. Óleo de soja.

\section{Introdução}

A contaminação das águas e solos por compostos oleosos provenientes das atividades industriais e urbanas vêm atingindo níveis insustentáveis, ocasionando uma preocupação crescente com relação aos sérios e irreversíveis impactos ambientais gerados.

O óleo comestível utilizado diariamente em residências, indústrias de alimentos e comércios é uma substância insolúvel em água e causa danos ambientais quando descartado diretamente no solo e nas redes de esgoto. Como o óleo apresenta menor densidade que a água, ele permanece na superfície do líquido, criando uma barreira que dificulta a entrada da luz e oxigênio, comprometendo a base da cadeia alimentar aquática, além de causar entupimento e transtornos no funcionamento das redes de esgoto. Quando em contato com o solo, ocasiona impermeabilidade, dificultando o escoamento das águas das chuvas e o acesso aos nutrientes necessários para a fauna e flora ali presentes (WILDNER; HILLING, 2012; THODE FILHO et al., 2013). A reparação deste solo para torná-lo novamente fértil e apto para cultivo é um processo oneroso e difícil (WILDNER; HILLING, 2012).

Diversas técnicas para remediação de ambientes contaminados vêm sendo aplicadas (TONINI; REZENDE; GRATIVOL, 2010; SOUZA; PENNA; OLIVEIRA, 2014), destacando-se os processos biológicos. Estes processos resultam principalmente num baixo custo de operação, assim como

\footnotetext{
${ }^{1}$ E-mail: tmcosta02@gmail.com

²E-mail: joaosperb@gmail.com

${ }^{3}$ E-mail: andre_zfc@hotmail.com

${ }^{4}$ E-mail: tatianibotelho27@gmail.com

${ }^{5}$ E-mail: thais.sell@hotmail.com

${ }^{6} \mathrm{E}$-mail: savio@furb.br

${ }^{7}$ E-mail: lorena@furb.br

FURB - Universidade Regional de Blumenau, Programa de Pós-Graduação em Engenharia Ambiental. CAMPUS ॥ Complexo Tecnológico. Rua São Paulo, 3250 - Itoupava Seca. CEP 89.030-000 - BLUMENAU - SC, Brasil
} 
ausência de subprodutos residuais e baixo consumo de energia (SOUZA; PENNA; OLIVEIRA, 2014). A biorremediação é uma estratégia de grande interesse para descontaminação de solos (BANAT, 1995; MANCERA-LOPEZ et al., 2008; BANAT et al., 2010; MACIEL et al., 2010) devido a eficácia destes processos (ISMAIL et al., 2013) fazendo uso de microrganismos com potencial de crescimento nestes ambientes contaminados.

Algumas espécies microbianas, isoladas de ambientes contendo alta concentração de lipídios têm atraído maior atenção para estudos em processos biotecnológicos (MACIEL et al., 2010). Isto é decorrente da capacidade de metabolizar e degradar parcialmente os compostos oleosos presentes nestes meios, proporcionando crescimento e síntese de distintas enzimas, como as lipases (triacilglicerol acilhidrolase, EC 3.1.1.3) e moléculas com características biossurfactantes (RIGAS et al., 2007; MARTINS; KALIL; COSTA, 2008; COLLA et al., 2010; ROVEDA; HEMKEMEIER; COLLA, 2010; CAMILIOS-NETO et al., 2011; SLIVINSKI et al., 2012; FLORCZAK et al., 2013).

$O$ uso de fungos filamentosos em processos de biorremediação tem sido reportado como eficaz, pelo aparato enzimático destes microrganismos e seu potencial para produção de biossurfactantes (MACIEL et al., 2013). Em geral, a síntese destes compostos pode ser decorrente de seu crescimento em meios que contêm lipídios como fonte de carbono (óleos vegetais, ácidos graxos), apresentando amplo patamar de aplicação industrial (JAEGER; REETZ, 1998; ROVEDA; HEMKEMEIER; COLLA, 2010; FLORCZAK et al., 2013).

Fungos do gênero Lentinula, Pleurotus, Geotrichum, Phanerochaete, Aspergillus e Rhizopus vêm apresentando resultados satisfatórios em estudos de remediação envolvendo efluente do processo de obtenção de azeite - proveniente da moagem de azeitona - e de efluentes domésticos (McNAMARA et al., 2008; KSHIRSAGAR, 2013). Fungos do gênero Aspergillus (KAMINI; MALA; PUVANAKRISHNAN, 1998; MAHADIK et al., 2002; COLLA et al., 2010; MARTINS; KALIL; COSTA, 2008), Penicillium (VARGAS et al., 2008; DI LUCCIO et al., 2004) e Rhizopus (RODRIGUES, 2011) apresentaram resultados positivos na síntese de lipases e biossurfactantes induzidos na presença de substratos oleosos.

Diversas são as formas de selecionar novos fungos filamentosos com potencial de degradação de óleos vegetais e síntese de biocompostos. Dentre elas está a análise de afinidade e crescimento destes microrganismos em cultivos contendo substratos oleosos. A velocidade de crescimento radial, em determinadas condições é uma medida intrínseca e específica de cada gênero e espécie de fungo, importante para estudos de tolerância ao meio avaliado (LUIZ, 2010), podendo ser utilizada como técnica complementar no estudo de identificação microbiana (MACHADO, 2006).

Desta forma, o presente trabalho teve como objetivo identificar fungos filamentosos isolados de ambientes hidrofóbicos e avaliar a influência do óleo vegetal virgem e residual no crescimento destes fungos, por meio de um estudo cinético em placas de Petri.

\section{Material e métodos}

\subsection{Estirpes fúngicas}

Os fungos filamentosos avaliados neste estudo foram anteriormente isolados de resíduos procedentes de uma refinaria de petróleo, localizada em Canoas (RS, Brasil) e de uma refinaria de óleo vegetal, localizada em Gaspar (SC, Brasil) (SPERB et al., 2015). Todos os fungos foram mantidos em placas de Petri contendo meio de cultivo ágar batata dextrose (BDA - Kasvi), sendo armazenados a temperatura de $4{ }^{\circ} \mathrm{C}$, com repique a cada 2 meses e reativados para uso nos testes de identificação fúngica e de crescimento micelial radial.

\subsection{Identificação das colônias isoladas}

As colônias de fungos filamentosos foram analisadas de acordo com seus aspectos macro e micro morfológicos e identificadas de acordo com o material disponibilizado no Atlas de micologia médica (ZAITZ; RUIZ; SOUZA, 2004) e no livro Micologia médica à luz dos autores contemporâneos (SIDRIM; ROCHA, 2004). As características macro morfológicas observadas foram: cor, textura, relevo e bordas das colônias. As características micro morfológicas foram realizadas analisando a 


\section{REA - Revista de estudos ambientais (Online) v.17, n. 2, p. 29-40, jul./dez. 2015}

morfologia das hifas fúngicas da colônia utilizando a técnica de microcultivo, adicionando fragmentos do fungo a uma porção quadrada de meio de cultivo BDA. Estas frações foram colocadas sobre lâminas cruzadas no interior de uma placa de Petri e recobertas com lamínulas. As placas foram encubadas por 5 dias a uma temperatura de $25^{\circ} \mathrm{C}$.

Após este período, as lamínulas contendo 0 micélio dos fungos foram coloridas com lactofenol azul de algodão e observadas em microscópico ótico (MOTIC, modelo Q7720AD). Imagens de microscopia eletrônica de varredura (MEV - VEJA 3 TESCAN) foram realizadas de modo a obter a confirmação da identidade dos fungos.

\subsection{Determinação da velocidade específica de crescimento micelial radial}

Os fungos isolados foram cultivados em placas de Petri previamente preparadas com meio de cultivo sólido descrito por Gabiatti Jr. et al. (2006), composto por: $\mathrm{NaNO}_{3} \quad\left(3 \mathrm{~g} \cdot \mathrm{L}^{-1}\right), \quad \mathrm{KH}_{2} \mathrm{PO}_{4} \quad\left(1 \mathrm{~g} \cdot \mathrm{L}^{-1}\right)$, $\mathrm{MgSO}_{4} \cdot 7 \mathrm{H}_{2} \mathrm{O}\left(0,5 \mathrm{~g} \cdot \mathrm{L}^{-1}\right)$, extrato de levedura $\left(1 \mathrm{~g} \cdot \mathrm{L}^{-1}\right)$, peptona $\left(0,3 \mathrm{~g} \cdot \mathrm{L}^{1}\right)$ e ágar bacteriológico $\left(20 \mathrm{~g}^{\cdot \mathrm{L}^{-1}}\right)$. Ao meio de cultivo foi adicionado $1 \%(\mathrm{v} / \mathrm{v})$ de fonte de carbono (óleo de soja virgem ou óleo de soja residual) para cada ensaio de crescimento micelial radial. O óleo de soja residual utilizado era proveniente do restaurante localizado no Centro de Ciências Tecnológicas da Universidade Regional de Blumenau e foi esterilizado em autoclave a $121{ }^{\circ} \mathrm{C}$ por 15 minutos juntamente com o meio de cultivo. $\mathrm{O}$ mesmo procedimento foi realizado para o óleo virgem.

O meio de cultivo sem adição de fonte de carbono (óleo de soja virgem ou óleo de soja residual) foi utilizado como branco, a fim de comparar os efeitos causados pela adição do óleo.

A medida e a avaliação do crescimento micelial radial foram determinadas adaptando os métodos descritos por Wheeler, Hurdman e Pitt (1991) e Laszlo e Silman (1993). Um disco de micélio de $9 \mathrm{~mm}$ de cada estirpe foi inserido no centro de uma placa de Petri contendo o meio de cultivo e encubados por 5 dias a uma temperatura de $25{ }^{\circ} \mathrm{C}$. O crescimento micelial radial foi mensurado utilizando um paquímetro, e medindo-se o diâmetro em duas posições perpendiculares, obtendo deste modo, o raio médio das colônias para cada 24 horas, conforme ilustrado na Figura 1.

Figura 1 - Determinação das medidas do diâmetro de crescimento radial em placas de Petri. Círculo central indica o disco de micélio de $9 \mathrm{~mm}$. As setas preta e branca apontam as medições inicial e final (após 5 dias) de crescimento do inóculo. Letras A, B, C e D correspondem aos eixos nos quais as leituras foram realizadas.

Fonte: Autores (2015)

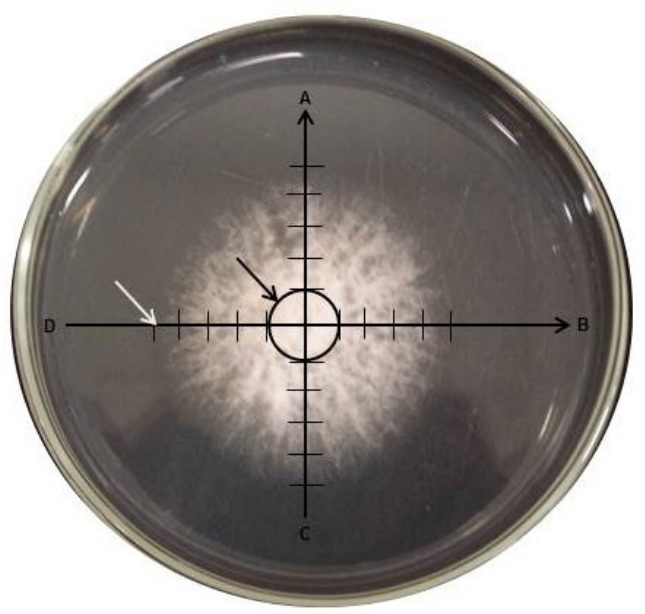

Os ensaios foram realizados em três repetições para cada estirpe e meio de cultivo. A velocidade específica de crescimento foi determinada para cada fungo em cada meio, de acordo com a equação 1. 


\section{REA - Revista de estudos ambientais (Online) v.17, n. 2, p. 29-40, jul./dez. 2015}

$$
\mu(t)=\frac{1}{D} \frac{d D}{d t}
$$

Onde $\mu(t)$ é a velocidade específica de crescimento $\left(\mathrm{h}^{-1}\right) ; t$ é o tempo (h) e $D$ é o diâmetro da colônia $(\mathrm{mm})$.

Os resultados das velocidades específicas de crescimento de cada fungo e meio de cultivo foram submetidos a análise de variância (ANOVA) e o teste de Tukey ao nível de $5 \%$ de significância para a comparação das médias. $O$ perfil radial e cinético de crescimento de cada fungo também foi avaliado para cada meio de cultivo.

\section{Resultados e discussão}

\subsection{Análise das características e identificação dos fungos isolados}

As características morfológicas de fungos filamentosos são normalmente descritas em dois níveis - macroscópico e microscópico. Com a macro morfologia é possível descrever características mais robustas como cores, texturas, relevos de pellets ou de micélios, que podem estar aglomerados ou livremente dispersos. Através da micro morfologia, é possível verificar propriedades mais específicas, como frequência de ramificação, dimensões de hifas e de segregação (McINTYRE et al., 2001).

Neste estudo, foi possível a identificação de 9 dos 24 fungos filamentosos testados, até o nível de gênero. Os fungos LEB 022, 024, 028 e 031 foram identificados como integrantes do gênero Penicillium; os fungos LEB 037, 040 e 044 do gênero Rhizopus; o fungo LEB 025 como Aspergillus e o fungo LEB 038 como Scopulariopsis. Os demais fungos não foram possíveis de serem identificados pelos métodos adotados, devido a dificuldade de observação das estruturas de reprodução. $\mathrm{Na}$ Figura 2 estão mostradas as microestruturas reprodutivas que possibilitaram a identificação ao nível de gênero.

Figura 2 - Fungos identificados como: (A) Aspergillus sp.; (B) Scopulariopsis sp.; (C) Rhizopus sp.; (D) Penicillium sp.
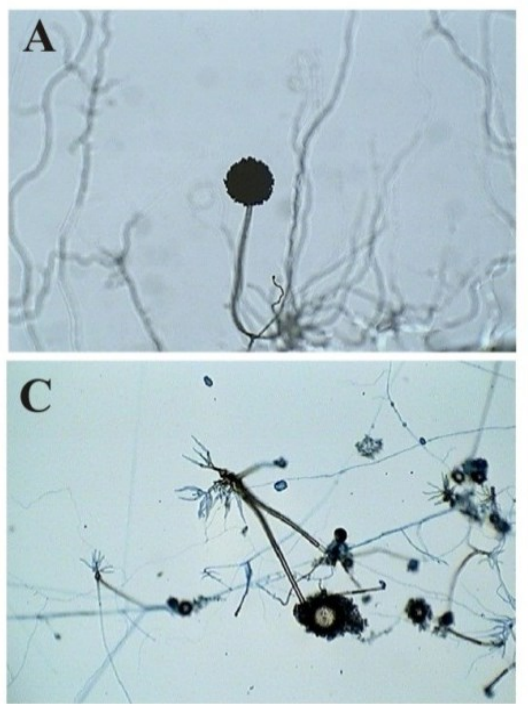

Fonte: Autores (2015)

O fungo LEB 025 teve sua espécie identificada como Aspergillus niger (Figura 3 A) em virtude do número de variáveis distintas macro e micro morfológicas dentre os isolados. Apresentou intensa coloração preta dos esporos e superfície rugosa no verso da colônia. Como características micro morfológicas, apresentou conídios com cabeças café-escuro ao preto, conídios
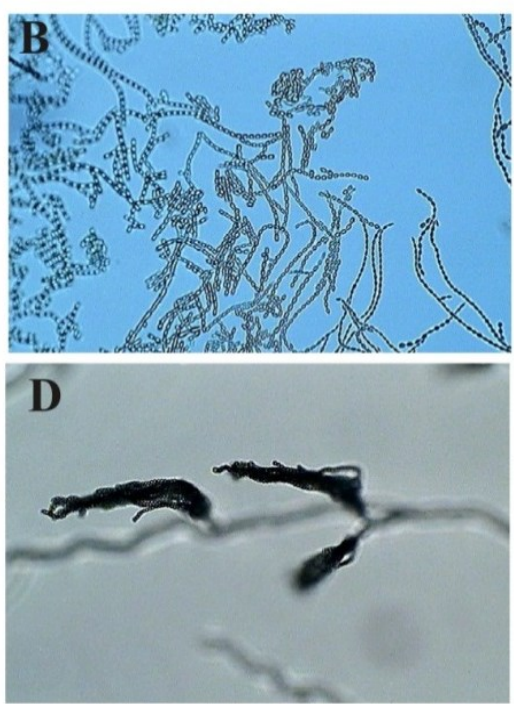

globulares, verrugosos e finamente enrugados (CRUZ, 2014), conforme observados nos ensaios de MEV (Figura 3 B). Entretanto, testes empregando técnicas de biologia molecular são importantes para corroborar a identificação da espécie obtida, bem como na identificação dos demais fungos não identificados. 


\section{REA - Revista de estudos ambientais (Online) v.17, n. 2, p. 29-40, jul./dez. 2015}

Figura 3 - (A) Características macro da colônia; (B) Microscopia eletrônica de varredura

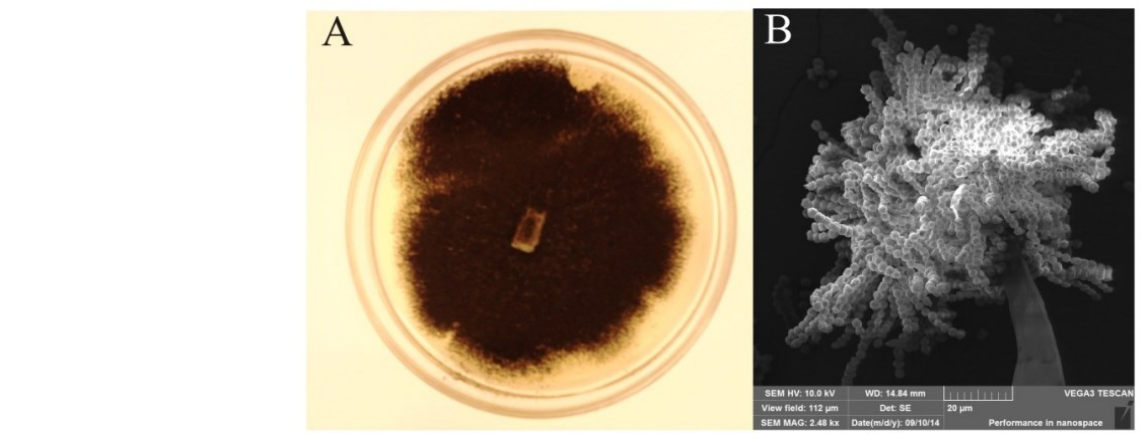

Fonte: Autores (2015)

Em trabalho apresentado por Faia (2011), fungos de gênero Aspergillus, Penicillium, Fusarium, Cladosporium dentre outros, foram identificados através de características macroscópicas (efetuando registros fotográficos) e microscópicas (microcultivo) em amostras de água antes e depois do processo de tratamento numa rede de distribuição predial. Aspergillus, Penicillium e Fusarium também foram identificados por Costa e Zanella (2012) em derivados de milho, utilizando as mesmas técnicas aplicadas neste estudo. Isto denota que as técnicas utilizadas são passíveis de serem utilizadas para identificação de fungos destes gêneros.

Estudos envolvendo fungos do gênero Aspergillus, Penicillium, Rhizopus e Scopulariopsis, vêm demonstrando a capacidade de crescimento e síntese de compostos como lipases (NAGY et al., 2006; CUZZI et al., 2011; KUMAR et al., 2012) e biossurfactantes quando na presença de substratos oleosos (MARTINS et al., 2008; CASTIGLIONI; BERTOLIN; COSTA, 2009) bem como em tratamentos e reutilização de águas residuais contendo óleo de oliva (McNAMARA et al., 2008).

\subsection{Determinação da velocidade específica de crescimento micelial radial}

$O$ efeito dos diferentes meios de cultura utilizados neste estudo (branco, óleo de soja virgem e óleo de soja residual) no crescimento micelial de fungos filamentosos foi avaliado. A partir dos ensaios em placa de Petri, a cinética e a velocidade específica de crescimento microbiano foram obtidos. Verificou-se que os meios de cultura testados são fatores determinantes no perfil de crescimento fúngico, visto que a alteração nestes meios altera significativamente 0 diâmetro das colônias.

Os resultados para as velocidades específicas máximas de crescimento $\left(\mu_{\max }\right)$ se encontram na Tabela 1, apresentados por fungos (sigla de denominação LEB e sua respectiva identificação), por locais de isolamento (resíduo do refino de óleo vegetal e resíduo do refino de petróleo) e pelo meio de cultura testado (branco, óleo de soja virgem e residual).

A $\mu_{\max }$ dos fungos nos diferentes meios de cultivo foi analisada por meio da ANOVA, sendo observado diferenças significativas entre os resultados obtidos para os diferentes fungos $(p<0,05)$. A comparação das médias da $\mu_{\max }$ por meio de teste de Tukey mostrou que os fungos LEB 025 (Aspergillus niger), 029, 033, 039, 040 (Rhizpous sp.) e 042 apresentaram resultados significativamente superiores $(p<$ 0,05 ) aos demais fungos quando cultivados em meio contendo óleo de soja residual.

O fungo LEB 029 apresentou a mais elevada velocidade específica de crescimento no óleo de soja residual dentre os fungos estudados, com rápida adaptação já nas primeiras horas de cultivo. Esse dado é importante para se estabelecer um menor tempo de cultivo dos fungos que apresentaram maior velocidade de crescimento radial (SCHUBER et al., 2012). No entanto, análises mais apuradas de identificação do fungo se fazem necessárias, de modo a obter maior conhecimento sobre o microrganismo em estudo.

Mudanças físicas (escurecimento, aumento de viscosidade) e químicas (hidrólise, oxidação) ocorrem no óleo durante o processo de fritura por imersão. Sanibal e Mancini-Filho (2002) avaliaram as mudanças na composição dos ácidos graxos do óleo de soja utilizados no processo de fritura. Eles observaram que o processo de fritura leva a 


\section{REA - Revista de estudos ambientais (Online) v.17, n. 2, p. 29-40, jul./dez. 2015}

uma diminuição na concentração dos ácidos graxos poliinsaturados, ocasionando um aumento proporcional dos ácidos graxos saturados e um aumento na formação dos ácidos graxos trans. Esta alteração na conformação do óleo pode ter contribuído para maior adaptação microbiana neste meio.

Tabela 1 - Fungos isolados e velocidade específica máxima de crescimento nos diferentes meios de cultura testados

\begin{tabular}{|c|c|c|c|c|}
\hline \multicolumn{5}{|c|}{ Resíduo do refino de óleo vegetal } \\
\hline \multirow{2}{*}{$\begin{array}{l}\text { Fungo } \\
\text { (LEB) }\end{array}$} & \multirow[b]{2}{*}{ Identificação } & \multicolumn{3}{|c|}{$\mu\left(\mathrm{h}^{-1}\right)$} \\
\hline & & Branco & $\begin{array}{c}\text { Óleo de soja } \\
\text { virgem }\end{array}$ & $\begin{array}{c}\text { Óleo de soja } \\
\text { residual }\end{array}$ \\
\hline 022 & Penicillium sp. & $0,015^{a} \pm 0,006$ & $0,012^{a} \pm 0,002$ & $0,036^{a} \pm 0,020$ \\
\hline 023 & $\mathrm{NI}$ & $0,022^{a} \pm 0,004$ & $0,023^{a} \pm 0,000$ & $0,027^{\mathrm{a}} \pm 0,004$ \\
\hline 024 & Penicillium sp. & $0,013^{a} \pm 0,001$ & $0,012^{a} \pm 0,001$ & $0,019^{a} \pm 0,006$ \\
\hline 025 & Aspergillus niger & $0,021^{a} \pm 0,001$ & $0,046^{a} \pm 0,007$ & $0,089^{b} \pm 0,027$ \\
\hline 026 & $\mathrm{NI}$ & $0,021^{a} \pm 0,000$ & $0,023^{a} \pm 0,002$ & $0,024^{a} \pm 0,005$ \\
\hline 027 & $\mathrm{NI}$ & $0,027^{a} \pm 0,001$ & $0,032^{\mathrm{a}} \pm 0,002$ & $0,055^{a} \pm 0,038$ \\
\hline 028 & Penicillium sp. & $0,012^{\mathrm{a}} \pm 0,004$ & $0,015^{a} \pm 0,005$ & $0,038^{a} \pm 0,032$ \\
\hline 044 & Rhizopus sp. & $0,076^{b} \pm 0,014$ & $0,140^{\mathrm{ab}} \pm 0,033$ & $0,208^{a} \pm 0,035$ \\
\hline 029 & $\mathrm{NI}$ & $0,064^{b} \pm 0,013$ & $0,138^{b} \pm 0,063$ & $0,403^{a} \pm 0,017$ \\
\hline 030 & $\mathrm{NI}$ & $0,017^{a} \pm 0,003$ & $0,023^{a} \pm 0,001$ & $0,023^{a} \pm 0,003$ \\
\hline 031 & Penicillium sp, & $0,013^{b} \pm 0,002$ & $0,016^{\mathrm{ab}} \pm 0,003$ & $0,026^{\mathrm{a}} \pm 0,006$ \\
\hline 032 & $\mathrm{NI}$ & $0,030^{a} \pm 0,001$ & $0,043^{a} \pm 0,013$ & $0,050^{\mathrm{a}} \pm 0,023$ \\
\hline 033 & $\mathrm{NI}$ & $0,120^{C} \pm 0,016$ & $0,167^{b} \pm 0,011$ & $0,270^{\mathrm{a}} \pm 0,024$ \\
\hline 034 & $\mathrm{NI}$ & $0,012^{a} \pm 0,002$ & $0,014^{a} \pm 0,005$ & $0,017^{\mathrm{a}} \pm 0,002$ \\
\hline 035 & $\mathrm{NI}$ & $0,012^{a} \pm 0,003$ & $0,008^{a} \pm 0,002$ & $0,013^{\mathrm{a}} \pm 0,003$ \\
\hline 036 & $\mathrm{NI}$ & $0,026^{a} \pm 0,006$ & $0,031^{a} \pm 0,003$ & $0,034^{a} \pm 0,001$ \\
\hline 037 & Rhizopus sp, & $0,042^{b} \pm 0,010$ & $0,059^{\mathrm{ab}} \pm 0,010$ & $0,087^{\mathrm{a}} \pm 0,013$ \\
\hline 038 & Scopulariopsis sp, & $0,021^{a} \pm 0,001$ & $0,027^{\mathrm{a}} \pm 0,004$ & $0,029^{a} \pm 0,007$ \\
\hline 039 & $\mathrm{NI}$ & $0,021^{b} \pm 0,000$ & $0,021^{b} \pm 0,002$ & $0,027^{\mathrm{a}} \pm 0,000$ \\
\hline 040 & Rhizopus sp, & $0,070^{b} \pm 0,019$ & $0,125^{b} \pm 0,020$ & $0,278^{a} \pm 0,057$ \\
\hline 041 & $\mathrm{NI}$ & $0,029^{a} \pm 0,001$ & $0,030^{a} \pm 0,001$ & $0,030^{\mathrm{a}} \pm 0,001$ \\
\hline 042 & $\mathrm{NI}$ & $0,029^{b} \pm 0,001$ & $0,033^{b} \pm 0,000$ & $0,040^{\mathrm{a}} \pm 0,003$ \\
\hline 043 & $\mathrm{NI}$ & $0,012^{a} \pm 0,002$ & $0,010^{a} \pm 0,001$ & $0,010^{\mathrm{a}} \pm 0,001$ \\
\hline 045 & $\mathrm{NI}$ & $0,026^{a} \pm 0,003$ & $0,070^{a} \pm 0,020$ & $0,052^{a} \pm 0,010$ \\
\hline
\end{tabular}

Letras iguais na mesma linha não apresentam diferença significativa ao nível de $5 \%$.

$\mathrm{NI}$ correspondem aos fungos não identificados

Fonte: Autores (2015)

Pode ser observado na Tabela 1, que a suplementação com óleo de soja virgem e residual nas quantidades utilizadas, não provocou inibição no crescimento dos fungos estudados em comparação ao meio em branco. Isto indica que os fungos testados apresentam capacidade de assimilar os constituintes presentes no óleo de soja estando esse na forma virgem ou residual. Os óleos vegetais apresentam cadeias de ácidos graxos menos complexas, quando comparados a outros tipos de óleos, sendo mais facilmente degradadas pelo metabolismo microbiano (GABIATTI Jr.et al., 2006).

Fungos do gênero Aspergillus isolados de solos contaminados com herbicidas triazínicos, apresentaram elevada capacidade de crescimento em meios contendo $50 \mathrm{ppm}$ de atrazine (COLLA et al., 2008), assim como em trabalhos apresentados por Vale et al. (2011), no qual o fungo Aspergillus niger, isolado de água residuária contendo óleo, compostos 
nitrogenados e metais pesados, se apresentou um microrganismo potencialmente aplicável para remoção de metais pesados de ambientes contaminados num estudo em placas. Não foram obtidos dados na literatura de crescimento em placas de Aspergillus em meios contendo óleo vegetal. No entanto, trabalhos apresentados por Colla et al. (2010), Martins, Kalil e Costa (2008) e Souza, Junior e Marra (2015), denotam a síntese de lipase e biossurfactante utilizando fungos Aspergillus e como fonte de carbono, o óleo de soja. Lima (2012) estudou a produção de biossurfactante pelos fungos Aspergillus ochraceus e Penicillium expansum utilizando óleo residual de fritura como fonte de carbono, encontrando resultados satisfatórios na biorremediação de áreas poluídas.

Enzimas lipolíticas extracelulares também foram sintetizadas por Rhizopus chinensis em meio contendo distintos óleos, dentre eles o óleo de soja (POGORI et al., 2008). O óleo de pequi, utilizado no crescimento em placas do fungo Rhizpous, não apresentou inibição quando submetido a alíquotas inferiores a $50 \mu \mathrm{L}$ em estudos realizados por Mendes et al. (2013). Isto denota o potencial de crescimento destes fungos em ambientes contaminados.

A maioria dos fungos não apresentou diferença significativa entre suas $\mu_{\max }$ nos meios suplementados com óleo virgem e no meio branco (Tabela 1). O fungo LEB 033 foi o único a apresentar diferença significativa de crescimento no óleo virgem quando comparado ao meio branco e ao meio suplementado com óleo residual. Também foi responsável pela maior velocidade específica de crescimento, $0,167 \mathrm{~h}^{-1}$ dentre os fungos quando suplementados com óleo de soja virgem.

As velocidades de crescimento específico e o crescimento micelial radial para cada fungo foram determinados por meio de análises cinéticas, nas condições deste estudo. A Figura 4 mostra os perfis cinéticos e radiais das estirpes LEB 022 (Penicillium sp.), 025 (Aspergillus niger), 032, 038 (Scopulariopsis sp), 042 e 045, que apresentaram os mais distintos perfis dentre os fungos estudados.

Pela análise do perfil cinético dos fungos mostrados na Figura 4, constata-se que a suplementação com o óleo de soja residual proporcionou indução no crescimento microbiano de LEB 022, 025, 032 e 042. O fungo LEB 022, identificado como Penicillium sp., apresentou elevada velocidade específica de crescimento em meio contendo óleo residual já nas primeiras 24 horas de cultivo, destacando-se frente ao óleo virgem e ao meio branco. Ao longo das 96 horas de cultivo, o fungo LEB 022 apresentou crescimento similar tanto no meio com óleo virgem como no meio branco.

O fungo Aspergillus niger LEB 025, apresentou crescimento induzido nos dois meios suplementados com as fontes de carbono, com preferência ao óleo residual, apresentando $\mu_{\max }$ de $0,089 \mathrm{~h}^{-1}$ e $0,046 \mathrm{~h}^{-1}$ para óleo de soja residual e virgem, respectivamente. Após 40 horas, as velocidades de crescimento nos meios suplementados tornaram-se inferiores ao meio branco para os fungos LEB 025 e 032, que apresentaram elevado crescimento nos meios suplementados já no início do cultivo.

Perfis similares ao fungo LEB 025 foram observados para os fungos LEB 029, 033, 040 e 044 (dados não mostrados). É provável que a rápida adaptação e assimilação dos nutrientes no início do processo nos meios suplementados com os óleos, tenham proporcionado um rápido crescimento, ocasionando uma redução dos nutrientes disponíveis ao longo do tempo. Já no meio branco, estes fungos necessitaram de maior tempo de adaptação. Tal resultado pode ser decorrente de restrições em termos de fonte de carbono no meio branco.

O fungo Scopulariopsis LEB 038, em meio com óleo residual, necessitou de um tempo maior de adaptação inicial frente ao óleo de soja virgem e ao meio branco. No entanto, após 10 horas, uma velocidade ascendente no meio com óleo residual foi verificada, mantendo-se superior aos demais meios de cultura até o final do cultivo. O tipo e a forma de preparo do alimento a ser frito contribui na modificação das propriedades do óleo, visto que, durante o processo de fritura dos alimentos, o óleo passa por uma série complexa de reações, modificando a qualidade funcional, sensorial e nutricional deste óleo (ANS; MATTOS; JORGE, 1999). Estas modificações podem proporcionar indução como também inibição no crescimento microbiano.

Para o fungo LEB 042 o óleo de soja virgem proporcionou maior dificuldade de adaptação, ocasionando baixo crescimento micelial radial dentre os meios testados. Já para LEB 045, este meio proporcionou estímulo no crescimento do fungo, quando comparado ao meio branco e suplementado com óleo residual. 
Figura 4 - Perfis de crescimento cinético e radial dos fungos LEB 022, 025, 032, 038, 042 e 045 Óleo de soja virgem; ....... Óleo de soja residual; _. . . Branco. Valores experimentais para:

- Óleo de soja virgem; $\Delta$ Óleo de soja residual; $\bullet$ Branco.
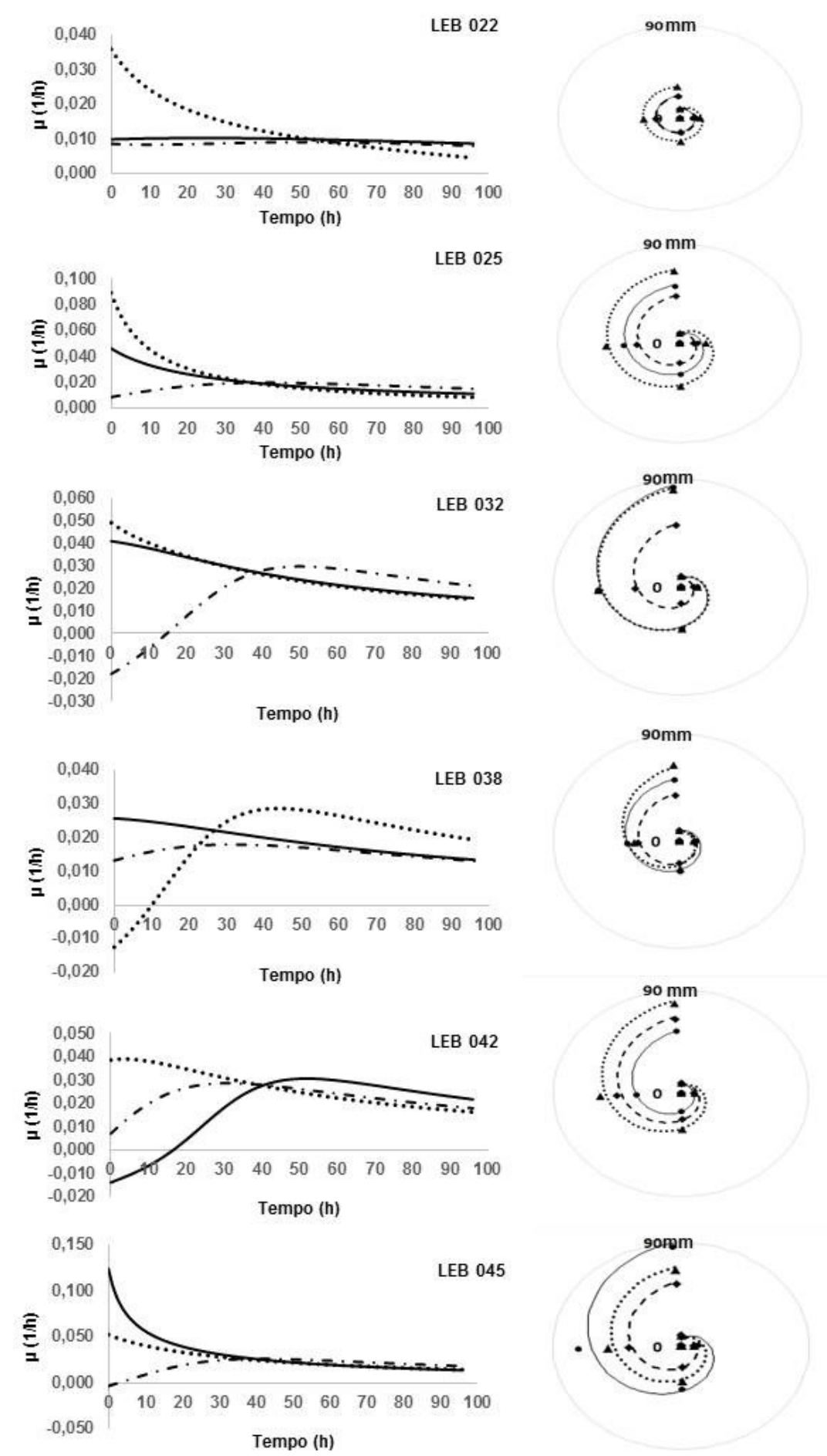

Fonte: Autores (2015)

A velocidade de crescimento microbiano é variável entre fungos e entre suas espécies. Fungos que possuem maiores e mais rápido crescimento das hifas acarretam maior formação de metabólitos secundários, viabilizando a produção em larga escala de um produto comercial (OLIVEIRA, 2008). A capacidade de assimilar mais rapidamente o óleo residual e as velocidades específicas máximas de 


\title{
REA - Revista de estudos ambientais (Online)
}

v.17, n. 2, p. 29-40, jul./dez. 2015

crescimento micelial apresentadas pelos fungos LEB 025, 029, 033, 039, 040, 042 e 044, denotam o potencial de adaptação e crescimento destes fungos num meio contaminado com este poluente.

A seleção de novos fungos produtores de enzimas é uma técnica de grande importância, principalmente em países que apresentam uma elevada biodiversidade como o Brasil, o que justifica a busca de fontes alternativas de novas cepas fúngicas para a produção de enzimas lipolíticas (SCHUBER et al., 2012). Óleos e gorduras vêm sendo apontados como principal fonte de carbono indutora na produção de lipases em processos fermentativos utilizando microrganismos (MURUCl, 2012). O uso de óleos residuais pode contribuir na redução do custo da matéria-prima utilizada para a produção enzimática, visto que, o óleo de fritura pode ser um indutor de crescimento microbiano.

Os fungos LEB 025 (Aspergillus niger), 029, 033, 039, 040 (Rhizopus sp.), 042 e 044 (Rhizopus sp.) selecionados neste estudo, podem vir a sintetizar resultados favoráveis de produção de enzima lipolítica, bem como ser testados em processos de biorremediação de ambientes contaminados com este poluente em estudos posteriores.

\section{Conclusões}

Neste estudo foi possível a identificação de 9 dos 24 fungos isolados em termos de gênero sendo um em nível de espécie, perante as técnicas macro e micro morfológicas utilizadas. Entretanto, o uso das técnicas de biologia molecular são necessárias para a identificação de todos os gêneros e espécies utilizadas neste trabalho. Análises das velocidades específicas de crescimento e de seus perfis cinéticos de crescimento mostraram que os isolados LEB 022 (Penicillium sp.), 025 (Aspergillus niger), 029, 033, 039, 040 (Rhizopus sp.), 042 e 044 (Rhizopus sp.) apresentaram melhores performances de crescimento em meio contendo óleo de soja residual quando comparado ao meio branco, com destaque para o fungo LEB 029. Isto denota que através de um estudo em placas, é possível selecionar, de forma primária, fungos que apresentam potencial de crescimento em ambientes contaminados com óleo de soja virgem ou residual. Isso possibilita a utilização destes fungos em novos estudos envolvendo uma possível aplicação em remediação de ambientes contaminados, bem como sua utilização para síntese de compostos de aplicação industrial.

\section{Evaluation of Radial Specific Growth Rate Fungus in Residual Vegetable Oil}

\begin{abstract}
The oily wastes amounts generated daily by different industries as well as their improper disposal in residential and natural environments have caused global concern due the extent of environmental damage caused by these pollutants. Different fungi have potential for growth when cultivated in environments containing oily substrates. The radial mycelial growth as well as the kinetics and specific growth rate of filamentous fungi isolated from wastes of vegetable and crude oil processing were evaluated on Petri dishes containing virgin or residual soybean oil. Among the 24 tested fungi, identification of the genus Penicillium, Rhizopus, Aspergillus and Scopulariopsis was done. Fungi LEB 025, 029, 033, 039, 040, 042 and 044 presented the maximum specific growth rates stimulated by the presence of residual soybean oil, with no growth inhibition for any strain studied. These results suggest that fungi on this study may have potential for uses on bioremediation of environments contaminated by residual oils as well as their use for compound synthesis such as lipolytic enzymes and biosurfactants.
\end{abstract}

Keywords: Identification; Kinetics; Soybean oil.

\section{Referências}

ANS, V. G.; MATTOS, E. S.; JORGE, N. Avaliação da qualidade dos óleos de fritura usados em restaurantes, lanchonetes e similares. Food Science and Technology, v.19, n.3,1999.
BANAT, I. M.; FRANZETTI, A.; GANDOLFI, I.; BESTETTI, G.; MARTINOTTI, M. G.; FRACCHIA, L.; SMYTH T. J.; MARCHANT, R. Microbial biosurfactants production, applications and future potential. Applied Microbiology and Biotechnology, v. 87, p. 427-444, 2010. 


\section{REA - Revista de estudos ambientais (Online) v.17, n. 2, p. 29-40, jul./dez. 2015}

BANAT, I. M. Biosurfactants production and possible used in microbial enhanced oil recovery and oil pollution remediation: a review. Bioresource Technology, v. 51, p.1-12, 1995.

CAMILIOS-NETO, D.; BUGAY, C.; SANTANAFILHO, A. P.; JOSLIN, T.; SOUZA, L. M.; SASSAKI, G. L.; MITCHELL, D. A.; KRIEGER, N. Production of rhamnolipids in solid-state cultivation using a mixture of sugarcane bagasse and corn bran supplement with glycerol and soybean oil. Applied Microbiology and Biotechnology, v. 89, p.1395-1403, 2011.

CASTIGLIONI, G. L.; BERTOLIN, T. E..; COSTA, J. A. V. Produção de biossurfactante por Aspergillus fumigatus utilizando resíduos agroindustriais como substrato. Química Nova, v.32, p.292-295, 2009.

COLLA, L. M.; PRIMAZ, A. L.; LIMA, M.; BERTOLIN, T. E.; COSTA, J. A. V. Isolamento e seleção de fungos para biorremediação a partir de solo contaminado com herbicidas triazínicos. Ciência e Agrotecnologia, v.32, p.809-813, 2008.

COLLA, L. M.; RIZZARDI, J.; PINTO, M. H.; REINEHR, C. O.; BERTOLIN, T. E.; COSTA, J. A. $V$. Simultaneous production of lipases and biosurfactants by submerged and solid-state bioprocess. Bioresource Technology, v.101, p.8308-8314, 2010.

COSTA, J. A. A.; ZANELLA, G. N. Identificação de fungos filamentosos em derivados de milho comercializados em Primavera do Leste - MT. Revista Brasileira de Farmácia, v.93, p.109-113, 2012.

CRUZ, R. Guia para el diagnóstico de laboratorio de enfermedad fúngica invasora por hongos filamentosos. Revista Chilena Infectol, v.31, p.173-179, 2014.

CUZZI, C.; LINK, S.; VILANI, A.; ONOFRE, S. B. Enzimas extracelulares produzidas por fungos endofíticos isolados de Baccharis Dracunculifolia D.C. (ASTERAECEAE). Global Science and Technology, v 4, n.2, p. 47-57, 2011.

Di LUCCIO, M.; CAPRA, F.; RIBEIRO, N. P.; VARGAS, G. D. L. P.; FREIRE, D. M. G.; De OLIVEIRA, D. Effect of Temperature, Moisture and Carbon Supplementation on lipase production by Solid State fermentation of Soy Cake by Penicillium simplicissimum. Applied Biochemistry and Biotechnology, v.113 - 116, p.173-180, 2004.

FAIA, A. M. Isolamento e identificação de fungos filamentosos e leveduras em alguns pontos de uma rede de distribuição de água. Dissertação (Mestrado em Biologia Celular e Biotecnologia). Universidade de Lisboa, Faculdade de Ciências, Portugal, 2011.
FLORCZAK, T.; DAROCH, M.; WILKINSON, M. C.; BIALKOWSKA, A.; BATES, A. D.; TURKIEWICZ, M.; IWANEJKO, L. A. Purification, characterization and expression in Saccharomyces cerevisiae of LipG7 an enantioselective, cold-adapted lipase from the Antarctic filamentous fungus Geomyces sp, P7 with unusual thermostability characteristics. Enzyme and Microbial Technology v.53, p.1824, 2013

GABIATTI Jr, C.; VENDRUSCOLO, F.; PIAIA, J. C. Z.; RODRIGUES, R. C.; DURRANT, L. R.; COSTA, J. A. V. Radial Growth Rate as a Tool for the Selection of Filamentous Fungi for Use in Bioremediation. Brazilian Archives of Biology and Technology, v.49, p.29-34, 2006.

ISMAIL, W.; AL-ROWAIHI, I. S.; AL-HUMAM, A. A.; HAMZA, R. Y.; EL NAYAL, A. M.; BOUOUDINA, $M$. Characterization of a lipopeptide biosurfactant produced by a crude-oilemulsifying Bacillus $\mathrm{sp}, \mathrm{I}-15$. International Biodeterioration \& Biodegradation, v. 84, p.168-178, 2013.

JAEGER, K. E.; REETZ, M. T. Microbial lipases form versatile tools for biotechnology. Trends in Biotechnology, v.16, p.396-403, 1998.

KAMINI, N. R.; MALA, J. G. S.; PUVANAKRISHNAN, R. Lipase production from Aspergillus niger by solid state fermentation using gingelly oil cake. Process Biochemistry, v.33, p.505-511, 1998.

KSHIRSAGAR, A. D.; Application of bioremediation processes for wastewater treatment using aquatic fungi. International Journal of Current Research, v.5, p.1737-1739, 2013.

KUMAR, S.; MATHUR, A.; SINGH, V.; NANDY, S.; KHARE S.K.; NEGI, S. Bioremediation of waste cooking oil using a novel lipase produced by Penicillium chrysogenum SNP5 grown in solid medium containing waste grease. Bioresource Technology, v. 120, p. 300-304, 2012.

LASZLO, J. A.; SILMAN, R. W.; Cellular automata simulations of fungal growth on solid substrates. Biotechnology Advances, v.11, p.621-633, 1993.

LIMA, B. M. Produção de biossurfactantes pelos fungos Aspergillus ochraceus e Penicillium expansum em fermentação semisólida utilizando resíduos agroindustriais como substrato. Dissertação (Mestrado em Microbiologia), Universidade Estadual Paulista “Júlio de Mesquita Filho" UNESP, São José do Rio Preto, 2012.

LUIZ, F. C. J. P. F. Identificação fenotípica e genotípica de fungos filamentosos isolados de talcos comerciais cosméticos. Dissertação 


\section{REA - Revista de estudos ambientais (Online) v.17, n. 2, p. 29-40, jul./dez. 2015}

(Mestrado em Ciências Farmacêuticas), Universidade Federal do Paraná, Curitiba, 2010.

MACHADO, A. P. S. Uso de técnicas de detecção rápidas de fungos filamentosos na água. Dissertação (Mestrado em Tecnologia do Ambiente), Escola de Engenharia da Universidade do Minho, Portugal, 2006.

MACIEL, C. C. S.; SOUZA, M. A.; GUSMÃO, N. B.; CAMPOS-TAKAKI, G. M. Produção de enzimas do sistema lignolítico por fungos filamentosos isolados de locais impactados por petroderivados. Exacta, v. 8, p. 299-305, 2010.

MACIEL, C. C. S.; SOUZA, C. S.; SILVA, P. A.; SOUZA, M. F. V. Q.; GUSMÃO, N. B. Cinética de degradação de querosene de aviação por Penicillium sp. através da bioestimulação. Revista Brasileira de Biociências, v.11, p.39-42, 2013.

MAHADIK, N. D.; PUNTAMBEKAR, U. S.; BASTAWDE, K. B.; KHIRE, J. M.; GOKHALE, D. V. Production of acidic lipase by Aspergillus niger in solid state fermentation. Process Biochemestry, v.38, p.715-721, 2002.

MANCERA-LOPEZ, M. E.; ESPARZA-GARCIA, F.; CHÁVEZ-GÓMEZ, B.; RODRÍGUEZVÁZQUEZ, R.; SAUCEDO-CASTAÑEDA, G.; BARRERA-CORTÉS, J. Bioremediation of an aged hydrocarbon-contaminated soil by a combined system of biostimulation bioaugmentation with filamentous fungi. International Biodeterioration \& Biodegradation, v 61, p.151-160, 2008.

MARTINS, V. G.; KALIL, S. J.; COSTA, J. A. V. Co-produção de lipase e biossurfactante em estado sólido para utilização em biorremediação de óleos vegetais e hidrocarbonetos. Quimica Nova, v.31, p.1942-1947, 2008

McINTYRE, M.; MÜLLER, C.; DYNESEN, J.; NIELSEN, J. Metabolic Engineering of the Morphology of Aspergillus. Advances in Biochemical Engineering/Biotechnology, v.73, p.103-128, 2001.

McNAMARA, C. J.; ANASTASIOU, C. C.; O'FLAHERTY, V.; MITCHELL, R. Bioremediation of olive wastewater. International Biodeterioration \& Biodegradation, v.61, p.127134, 2008.

MENDES, B. L.; ZANATTO, I. B.; RIBEIRO, A. S.; PASTORELLO, M.; BONALDO, S. M. Influência do óleo de pequi no crescimento micelial e esporulação de Colletotrichum musae e crescimento micelial de Rhizopus sp. in vitro. Scientific Eletronic Archives, v.3, p.20-23, 2013.

MURUCI, L. N. M. Produção e caracterização de lipase de Aspergillus niger obtida por fermentação no estado sólido utilizando resíduos da agroindústria. 2012. Tese
(Doutorado em Ciências) - Universidade Federal Rural do Rio de Janeiro, Seropédica, RJ, 2012.

NAGY, V.; TÖKE, E. R.; KEONG, L. C.; SZATZKER, G.; IBRAHIM, D.; OMAR, I. C.; SZAKÁCS, G.; POPPE, L. Kinetic resolutions with novel, highly enantioselective fungal lipases produced by solid state fermentation. Journal of Molecular Catalisys B: Enzymatic, v. 39, p. 141148, 2006.

OLIVEIRA, I. M. Aspectos biológicos do fungo entomopatogênico Aschersonia sp. cultivado em diferentes meios de cultura. Dissertação (Mestrado em Entomologia Agrícola) Universidade Federal de Lavras, Minas Gerais, 2008.

POGORI, N.; CHEIKHYOUSSEF, A.; XU, Y.; WANG, D. Production and Biochemical characterizations of an extracellular lipase from Rhizopus chinensis CCTCC M201021. Biotechnology, v.7, p.710-717, 2008.

RIGAS, F.; PAPADOPOULOU, K.; DRITSA, V.; DOULIA, D. Bioremediation of a soil contaminated by lindane utilizing the fungus Ganoderma australe via response surface methodology. Journal of Hazardous Materials, v.140, p. 325332, 2007.

RODRIGUES, C. Seleção de fungos produtores de lipases a partir de resíduos oleosos derivados do saneamento ambiental. 2011. Tese (Doutorado em Engenharia Ambiental) Universidade Federal do Espírito Santo, Vitória, 2011.

ROVEDA, M.; HEMKEMEIER, M.; COLLA, L. M. Avaliação da produção de lipases por diferentes cepas de microrganismos isolados em efluentes de laticínios por fermentação submersa. Food Science and Technology (Campinas), v.30, p.126-131, 2010.

SANIBAL, E. A. A.; MANCINI FILHO, J. Alterações físicas, químicas e nutricionais de óleos submetidos ao processo de fritura. Caderno de Tecnologia de Alimentos e Bebidas, p.4754, 2002.

SCHUBER, L. C. L.; CRUZ, M. C. R.; BONFIM, T. M. B.; BRAND, D.; PASTORE, G. M.; ALMEIDA, M. M. Isolamento e seleção de fungos produtores de $B$-Galactosidase. Evidência, v.12, p.19-40, 2012.

SIDRIM, J. J. C.; ROCHA, M. F. G. Micologia médica à luz de autores contemporâneos. Guanabara Koogan. Rio de Janeiro, 388 p., 2004.

SLIVINSKI, C. T.; MALLMANN, E.; ARAÚJO, J. M.; MITCHELL, D. A.; KRIEGER, N. Production of surfactin by Bacillus pumilus UFPEDA 448 in solid-state fermentation using a medium based on 


\section{REA - Revista de estudos ambientais (Online) v.17, n. 2, p. 29-40, jul./dez. 2015}

okara with sugarcane bagasse as a bulking agent. Process Biochemistry, v.47, p.1848-1855, 2012.

SOUZA, A. T.; JUNIOR, W. F. S.; MARRA, B. M. Produção de lipase por Aspergillus niger utilizando óleo de soja e açúcar comercial como fonte de carbono. Revista de Ciências Farmacêuticas Básica e Aplicada, v.36, 2015.

SOUZA, E. C.; PENNA, T. C. V.; OLIVEIRA, R. P. S. Biosurfactant-enhanced hydrocarbon bioremediation: An overview. International Biodeterioration \& Biodegradation, v.89, p.8894, 2014.

SPERB, J. G. C.; COSTA, T. M.; VAZ, D. A.; VALLE, J. A. B.; VALLE, R. C. S. C.; TAVARES, L. B. B. Avaliação qualitativa da produção de lipase e biossurfactantes por fungos isolados de resíduos oleosos. Engevista, v.17, p.385-397, 2015.

THODE FILHO, S.; SANTOS, A. S. S.; ALMEIDA, T. M.; SILVA, E. R. Tecnologia ambiental aplicada ao gerenciamento e processamento do óleo vegetal residual no estado do Rio de Janeiro. Revista Eletrônica em Gestão, Educação e Tecnologia Ambiental - REGET. v.15, p.30263035, 2013

TONINI, R. M. C. W.; REZENDE, C. E.; GRATIVOL, A. D. Degradação e bioremediação de compostos do petróleo por bactérias: Revisão, Oecologia Austrealis, v.14, p.1027-1035, 2010.

VARGAS, G. D. L. P.; TREICHEL, H.; De OLIVEIRA, D.; BENETI, S. C.; FREIRE, D. M. G.; Di LUCCIO, M. Optimization of lipase production by Penicillium simplicissimum in soybean meal. Journal of Chemical Technology and Biotechnology, v.83, p.47-54, 2008.

VALE, M. S.; ABREU, K. V.; GOUVEIA, S. T.; LEITÃO, R. C.; SANTAELLA, S. T. Efeito da toxicidade de $\mathrm{Cr}$ (VI) e $\mathrm{Zn}$ (II) no crescimento do fungo filamentoso Aspergillus niger isolado de efluente industrial. Engenharia Sanitária e Ambiental, v.16, p.237-244, 2011.

WHEELER, K. A.; HURDMAN, B. F.; PITT, J. I. Influence of $\mathrm{pH}$ on the growth of some toxigenic species of Aspergillus, Penicillium and Fusarium, International Journal of Food Microbiology, v.12, p.141-150, 1991.

WILDNER, L. B. A.; HILLING, C. Reciclagem de óleo comestível e fabricação de sabão como instrumentos de educação ambiental. Revista Eletrônica em Gestão, Educação e Tecnologia Ambiental. REGET/UFSM, v.5, p.813-824, 2012.

ZAITZ, C.; RUIZ, L. R. B.; SOUZA, V. M. Atlas de micologia médica: diagnóstico laboratorial. Medsi, $2^{\circ}$ ed. Rio de Janeiro, 167 p., 2004.

\section{Agradecimentos}

Esta pesquisa foi apoiada financeiramente pela Coordenação de Aperfeiçoamento de Pessoal de Nível Superior (CAPES, Brasil) e pelo Conselho Nacional de Desenvolvimento Científico e Tecnológico (CNPq, Brasil) pelas bolsas de estudos concedidas. 